\title{
GCR Neon Isotopic Abundances: Comparison with Wolf-Rayet Star Models and Meteoritic Abundances
}

\author{
W.R. Binns* , M.E. Wiedenbeck ${ }^{\dagger}$, E.R. Christian ${ }^{* *}$, A.C. Cummings ${ }^{\ddagger}$, J.S. George ${ }^{\ddagger}$, M.H. Israel* ${ }^{*}$, \\ R.A. Leske ${ }^{\ddagger}$, R.A. Mewaldt ${ }^{\ddagger}$, E.C. Stone ${ }^{\ddagger}$, T.T. von Rosenvinge ${ }^{* *}$, and N.E. Yanasak ${ }^{\ddagger}$ \\ * Washington University, St. Louis, MO 63130 USA \\ ${ }^{\dagger}$ Jet Propulsion Laboratory, California Institute of Technology, Pasadena, CA 91109 USA \\ ${ }^{* *}$ NASA/Goddard Space Flight Center, Greenbelt, MD 20771 USA \\ ${ }^{\ddagger}$ California Institute of Technology, Pasadena, CA 91125 USA
}

\begin{abstract}
Measurements of the neon isotopic abundances from the ACE-CRIS experiment are presented. These abundances have been obtained in seven energy intervals over the energy range of $\sim 80 \leq \mathrm{E} \leq 280 \mathrm{MeV} / \mathrm{nucleon}$. The ${ }^{22} \mathrm{Ne} /{ }^{20} \mathrm{Ne}$ source ratio is derived using the measured ${ }^{21} \mathrm{Ne} /{ }^{20} \mathrm{Ne}$ abundance as a "tracer" of secondary production of the neon isotopes. We find that the ${ }^{22} \mathrm{Ne} /{ }^{20} \mathrm{Ne}$ abundance ratio at the cosmic-ray source is a factor of $5.0 \pm 0.2$ greater than in the solar wind. The GCR ${ }^{22} \mathrm{Ne} /{ }^{20} \mathrm{Ne}$ ratio is also shown to be considerably larger than that found in anomalous cosmic rays, solar energetic particles, most meteoritic samples of matter, and interplanetary dust particles. Recent two-component Wolf-Rayet models provide predictions for the ${ }^{22} \mathrm{Ne} /{ }^{20} \mathrm{Ne}$ ratio and other isotope ratios. Comparison of the CRIS neon, iron, and nickel isotopic source abundance ratios with predictions indicate possible enhanced abundances of some neutron-rich nuclides that are expected to accompany the ${ }^{22} \mathrm{Ne}$ excess.
\end{abstract}

\section{Introduction}

The abundance ratio of ${ }^{22} \mathrm{Ne} /{ }^{20} \mathrm{Ne}$ in the galactic cosmic rays (GCR) is well known to be high compared to the solar wind (SW) [1]. For neon, the solar wind (SW) is believed to best represent the composition of the pre-solar nebula [2]. The GCR neon isotopic abundances were measured first by a balloon-borne experiment [3]. Subsequent satellite experiments on IMP-7 [4], ISEE-3 [5,6], Voyager [7], Ulysses [8], and CRRES [9] verified this overabundance with more precise measurements.

Woosley and Weaver [10] suggested that this overabundance could be explained by a model in which the synthesis of neutron-rich isotopes in massive stars is directly proportional to their initial metallicity (fraction of elements heavier than $\mathrm{He}$ ). Cosmic rays originating in a region of the Galaxy with metallicity that is higher than the Solar System could produce an overabundance of ${ }^{22} \mathrm{Ne} /{ }^{20} \mathrm{Ne}$. However, this should also lead to an overabundance of the neutron-rich isotopes of $\mathrm{Mg}$ and $\mathrm{Si}$, which has not been observed [8]. Galactic chemical evolution might also play a role in the ${ }^{22} \mathrm{Ne} /{ }^{20} \mathrm{Ne}$ overabundance, since cosmic rays may represent a more recent sample of matter than solar system abundances [11].

Olive and Schramm [12] suggested that an alternative to the conclusion that the GCR ${ }^{22} \mathrm{Ne} /{ }^{20} \mathrm{Ne}$ ratio is anomalously high is that the Solar System could instead be anomalously low. Supernovae in the near vicinity of the pre-solar nebula could have injected large amounts of ${ }^{20} \mathrm{Ne}$ and other $\alpha$ particle nuclei shortly before the solar system formed, thus making the solar system anomalous rather than the GCRs.

Cassé and Paul [13] first suggested that the ${ }^{22} \mathrm{Ne} /{ }^{20} \mathrm{Ne}$ overabundance could be due to the injection and preacceleration of Wolf-Rayet (WR) star material that is mixed into interstellar medium to form the cosmic-ray source material. Soutoul and Legrain [14,15] have developed a diffusion model in which GCRs come preferentially from the inner Galaxy. The assumptions in this model are that 1) there is a gradient in the cosmic-ray density as a function of galacto-centric radius and that the density increases as one moves nearer the galactic center, 2) there is a larger gradient in the density of WR stars than for $\mathrm{O}$ stars as the galactocentric radius decreases [16], and 3) that these WR stars enrich the Galaxy locally in ${ }^{22} \mathrm{Ne}[17,18]$. Mewaldt [19] reviews in greater detail the above models that predate his paper.

In this paper we present measurements of the neon isotopic abundances obtained by the Cosmic Ray Isotope Spectrometer (CRIS) instrument on the Advanced Composition Explorer (ACE) spacecraft [20]. The ${ }^{22} \mathrm{Ne} /{ }^{20} \mathrm{Ne}$ ratio has been measured as a function of energy over the energy range $77<\mathrm{E} / \mathrm{M}<278 \mathrm{MeV} /$ nucleon. We derive the ${ }^{22} \mathrm{Ne} /{ }^{20} \mathrm{Ne}$ source ratio using our measurements of ${ }^{21} \mathrm{Ne},{ }^{19} \mathrm{~F}$, and ${ }^{17} \mathrm{O}$ abundances as "tracers" [21] of secondary production of the neon isotopes. 

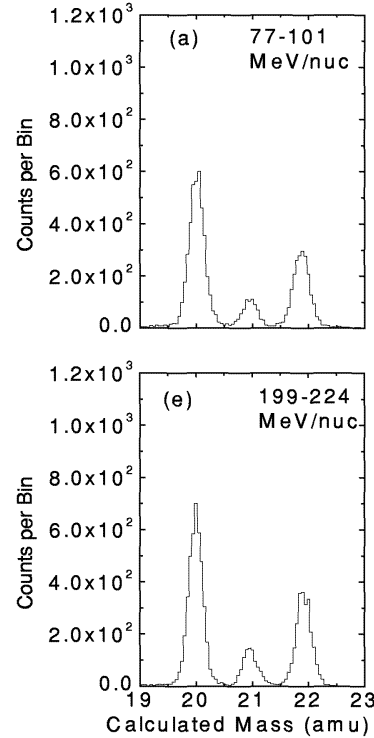
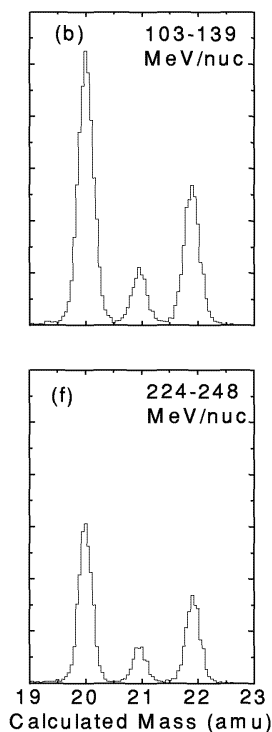
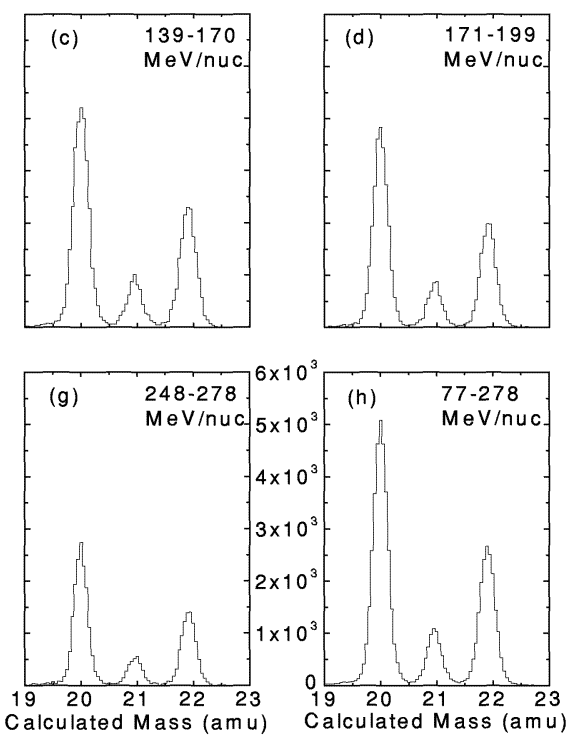

Figure 1-- Mass histograms of neon events in 7 energy bins (a-g). Figure $1 \mathrm{~h}$ is the sum over all the energy intervals [22].

\section{Measurements}

The CRIS neon data are plotted in Figure 1a-g for events in 7 energy bins spanning the energy range of $77<\mathrm{E} / \mathrm{M}<278 \mathrm{MeV} /$ nucleon. The sum of all events is shown in Figure 1h [22]. These events were collected from Dec. 4, 1997 through July 2, 2000 and are a selected, highresolution data set. Events included in this analysis had trajectory angles $\leq 25^{\circ}$ relative to the normal to the detector surfaces. Particles that stopped within $500 \mu \mathrm{m}$ of the one surface of each silicon wafer having a dead layer were excluded from this analysis. The numbers of events selected corresponds to $\sim 40 \%$ of the good events exclusive of these cuts. The rms mass resolution is $0.10 \mathrm{amu}$ for events plotted in Figure 1, which is sufficiently good so that there is only a slight overlap of the particle distributions for adjacent masses. Mass cuts have therefore been taken at the minima between peaks and the events within the cuts counted to obtain abundances. In Figure 1, the data plotted consist of $6.0 \times 10^{4}$ neon events. With the statistical accuracy of the CRIS data, it is possible, for the first time, to study the possible energy dependence of the ${ }^{22} \mathrm{Ne} /{ }^{20} \mathrm{Ne}$ ratio with high precision.

The ${ }^{22} \mathrm{Ne} /{ }^{20} \mathrm{Ne}$ and ${ }^{21} \mathrm{Ne} /{ }^{20} \mathrm{Ne}$ ratios measured by CRIS are plotted in Figure 2 as a function of energy. Corrections for energy spectra differences and nuclear interaction losses among the different isotopes have been made. The spectral dependence correction is $\sim 7.5 \%$ for the ${ }^{22} \mathrm{Ne} /{ }^{20} \mathrm{Ne}$ ratio and $\sim 3.7 \%$ for the ${ }^{21} \mathrm{Ne} /{ }^{20} \mathrm{Ne}$ ratio. The interaction correction is $<1 \%$ for both ratios. As can be seen in Figure 2, the GCR ${ }^{22} \mathrm{Ne} /{ }^{20} \mathrm{Ne}$ ratio is approximately constant with a small decrease toward lower energies. A similar behavior is observed for ${ }^{21} \mathrm{Ne} /{ }^{20} \mathrm{Ne}$, which also shows a roughly constant ratio with a slight decline for lower energies.
The ${ }^{21} \mathrm{Ne} /{ }^{20} \mathrm{Ne}$ ratio in the solar wind is low, $2.4 \times 10^{-3}$ [1]. Because of the much higher abundance of ${ }^{21} \mathrm{Ne}$ in GCRs (higher by two orders of magnitude) it is believed to be almost entirely secondary (i.e., resulting from nuclear interactions of primary cosmic rays as they propagate through the interstellar medium). This makes ${ }^{21} \mathrm{Ne}$ suitable for use as a "tracer" isotope, with mass near that of ${ }^{20} \mathrm{Ne}$ and ${ }^{22} \mathrm{Ne}$, for which the measured abundance can be scaled to estimate the fractions of ${ }^{22} \mathrm{Ne}$ and ${ }^{20} \mathrm{Ne}$ that are secondary [21].

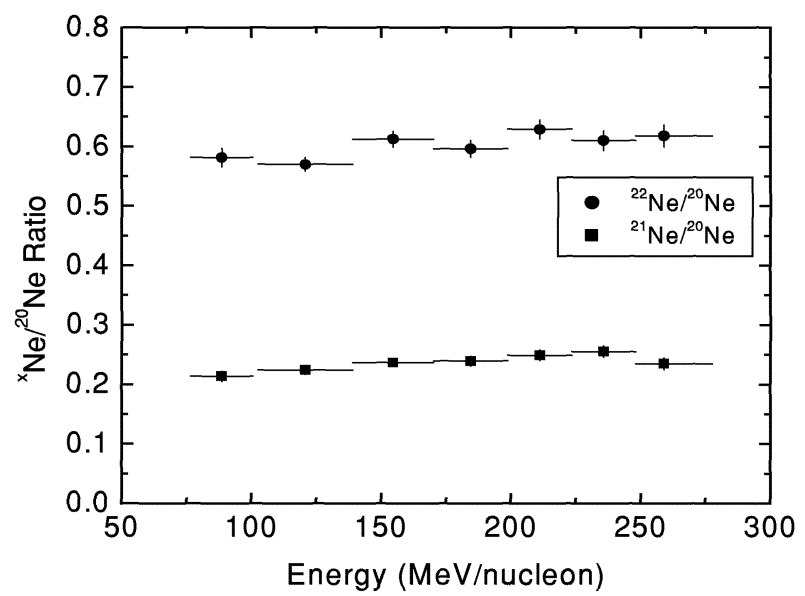

Figure 2-Plot of ${ }^{22} \mathrm{Ne} /{ }^{20} \mathrm{Ne}$ (circles) and ${ }^{21} \mathrm{Ne} /{ }^{20} \mathrm{Ne}$ (squares) ratios as a function of energy. In some cases the vertical error bars are smaller than the plotted point [22].

The CRIS $\mathrm{Ne}$ isotopic ratios averaged over the full energy range (taken from Fig $1 \mathrm{~h}$ ) have been compared with measurements made by other experiments [22]. The ratios reported from those experiments [5,7,8,9] have been adjusted to correspond to the approximate CRIS modulation level ( $500 \mathrm{MV})$. The CRIS measurement has smaller 
statistical uncertainties than previous experiments, and the agreement is generally good, although the Voyager ${ }^{22} \mathrm{Ne} /{ }^{20} \mathrm{Ne}$ value is somewhat lower than our ACE-CRIS ratio.

The measured ratio of ${ }^{22} \mathrm{Ne} /{ }^{20} \mathrm{Ne}$ has been corrected for secondary production during propagation using the tracer method. Source abundances were also obtained using ${ }^{19} \mathrm{~F}$ and ${ }^{17} \mathrm{O}$ secondary tracers. The ${ }^{22} \mathrm{Ne} /{ }^{20} \mathrm{Ne}$ galactic cosmic ray source ratios obtained using ${ }^{21} \mathrm{Ne},{ }^{19} \mathrm{~F}$, and ${ }^{17} \mathrm{O}$ as tracers were $0.350 \pm 0.004, \quad 0.375 \pm 0.003$, and $0.375 \pm .004$ respectively $[22,23]$. By averaging these three estimates, we obtain a "best" source abundance ratio of $0.366 \pm 0.006$ (stat.) \pm 0.014 (syst.) for ${ }^{22} \mathrm{Ne} /{ }^{20} \mathrm{Ne}$. The first quoted uncertainty in the abundance ratio is the statistical error, which takes into account both the measurement of ${ }^{22} \mathrm{Ne} /{ }^{20} \mathrm{Ne}$ and the uncertainty in the secondary correction associated with the statistics of the tracer isotope measurements. The second uncertainty is the systematic uncertainty estimate, calculated using the "sample standard deviation", obtained from the three tracer isotope estimates. In Figure 3 we plot the CRIS "best" value. The plotted error bar is the quadratic sum of the statistical and systematic uncertainties. It should be noted that the differing source ratio estimates from the three different tracer isotopes and the derived systematic uncertainties are comparable to those expected from nuclear cross-section uncertainties.

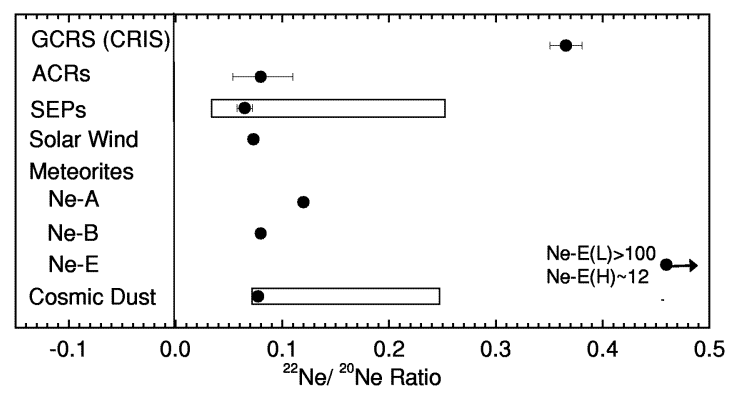

Fig. 3. The CRIS ${ }^{22} \mathrm{Ne} /{ }^{20} \mathrm{Ne}$ source abundance for GCRs are compared to Anomalous Cosmic Rays (ACRs) [24,25], solar wind [1,2], Solar Energetic Particles (SEPs) [26], meteoritic abundances [27], and cosmic dust (also called interplanetary dust particles or IDPs) [28]. The plotted error bar for CRIS is the quadratic sum of the statistical and systematic uncertainties (see text). The open bar for the SEP point represents the range of values measured in 11 solar events, while the data point and its uncertainty indicate the preliminary value deduced for the SEP source after accounting for the observed fractionation (Leske, private communication). Likewise the point plotted for Cosmic Dust is the average value obtained for 29 IDPs. The solid horizontal bar represents the spread in ratios for 27 of the particles measured, and the dotted horizontal bar the spread from the two remaining grains [28].

\section{Discussion}

Neon differs from most other elements in that it is observed to have a number of separate populations in "cosmic" matter with widely differing isotopic compositions.
Solar Wind--The solar wind is generally taken to give the best estimate of most isotopic ratios of noble gases including the Neon isotopes [2] in the presolar nebula. It is a sample of the solar corona and its isotopic composition is relatively stable on time scales comparable to the solar cycle. In Figure 3 the solar wind ${ }^{22} \mathrm{Ne} /{ }^{20} \mathrm{Ne}$ ratio is plotted $[1,2]$.

Solar Energetic Particles (SEPS)--SEPs are believed to be a sample of the outer corona (gradual events) and the lower corona (impulsive events) [30]. A larger variation is observed from event-to-event than is the case for the solar wind. Although there are large event-to-event variations the average is close to the solar wind value. In Figure 3, the SEP neon for 11 different events is shown as a horizontal bar that gives the range of measured ${ }^{22} \mathrm{Ne} /{ }^{20} \mathrm{Ne}$ ratios [26]. The single data point included in the bar indicates the preliminary value deduced for the SEP source after accounting for the observed fractionation (Leske, private communication).

Anomalous Cosmic Rays (ACRs)_ACRs result from neutral atoms in the very local interstellar medium drifting into the heliosphere where they are ionized by solar UV or charge exchange with the solar wind. They are then swept out to the solar wind termination shock where they are accelerated to energies of typically tens of $\mathrm{MeV}$ [31]. The $\mathrm{ACR}{ }^{22} \mathrm{Ne} /{ }^{20} \mathrm{Ne}$ abundance ratio $[25,23]$ is plotted in Figure 3.

Meteorites-The Ne-A component in meteorites is found in carbonaceous chondrites and may contain presolar components [32]. Ne-B is believed to result from solar wind [1] and very low-energy SEP implantation in grains [33] since it has a ${ }^{22} \mathrm{Ne} /{ }^{20} \mathrm{Ne}$ ratio very similar to that of contemporary measurements of the solar wind. Ne-E, which is found in $\mathrm{SiC}$ and graphite grains, is mostly ${ }^{22} \mathrm{Ne}$ [27], and probably comes from He burning asymptotic giant branch (AGB) stars and the decay of ${ }^{22} \mathrm{Na}$ produced in supernovae or novae [29,35]. Ne-E is the only population found in meteorites that has a ${ }^{22} \mathrm{Ne} /{ }^{20} \mathrm{Ne}$ ratio greater than that found for GCRs. The abundance ratios of these meteoritic components are plotted in Figure 3.

Cosmic Dust-The sources of cosmic dust (or interplanetary dust particles; IDPs) are believed to be asteroid belt and cometary dust material. As such they represent primitive matter from both the inner and outer solar system. The world inventory of cosmic dust particles consists of several thousand particles (as of the year 2000) and Kehm [28] presents the ${ }^{22} \mathrm{Ne} /{ }^{20} \mathrm{Ne}$ ratio for 29 of these particles. In Figure 3, we plot the mean value as a single data point with the solid horizontal bar giving the range of ratios for 27 of the grains and the dotted horizontal bar giving the range of the remaining two grains [28].

The GCR source ${ }^{22} \mathrm{Ne} /{ }^{20} \mathrm{Ne}$ abundance ratio is $5.0 \pm 0.2$ times greater than the $\mathrm{SW}$ value of 0.073 . The large enhancement in ${ }^{22} \mathrm{Ne} /{ }^{20} \mathrm{Ne}$ for GCRs compared with ACRs, which show good agreement with solar wind abundances is striking. This enhancement is also seen in the ACE-SIS data [25] and in the SAMPEX results [24]. If the ACR ratio is representative of the general ISM, then there is apparently a large overabundance of high-energy ${ }^{22} \mathrm{Ne}$ cosmic rays 
relative to ${ }^{20} \mathrm{Ne}$ compared to low energy matter (i.e., gas). We note that it is not presently known to what extent the ACRs actually represent the general ISM. It should also be noted that the solar system may not have formed in the local ISM. It has been suggested that the Sun may have formed $\sim 2 \mathrm{kpc}$ closer to the galactic center than our present galactocentric radius of $8.5 \mathrm{kpc}$, based on the higher metallicity of the Sun relative to that of nearby stars [34].

Wolf-Rayet Stars--The overabundance of ${ }^{22} \mathrm{Ne}$ in GCRs may be a result of the acceleration of WR star material as first suggested by Cassé and Paul [13]. It has been estimated that $\sim 2 \times 10^{-5}$ solar masses of material per year is ejected from a typical WR star in high velocity winds [18, 37]. There are two dominant phases of WR stars, the WC and $\mathrm{WN}$ phases. Large quantities of He-burning material rich in ${ }^{22} \mathrm{Ne}$ are expelled from the stars when they are in the WC phase, resulting in greatly enhanced ${ }^{22} \mathrm{Ne} /{ }^{20} \mathrm{Ne}$ ratios. In the WN phase of WR stars, CNO cycling dominates with the resultant production of high ${ }^{13} \mathrm{C} /{ }^{12} \mathrm{C}$ and ${ }^{14} \mathrm{~N} /{ }^{16} \mathrm{O}$ ratios, but no enhancement in the ${ }^{22} \mathrm{Ne} /{ }^{20} \mathrm{Ne}$ ratio $[18,36]$. It is thought that the pre-supernova WR winds might be swept up and accelerated by nearby supernovae or by the supernova shock from the WR star that ejected the material in the first place, without substantial mixing into the ambient ISM.

Recent modeling calculations by Meynet et al. [38] of the abundances of isotopes ejected from the surface of WR stars in the high velocity winds have incorporated the lower WR mass loss rates, updated nuclear reaction rates, and extended the reaction networks included in the models. Additionally, they have considered the effects of rotation, which has a strong effect on isotopic production. Their model is a two-component model that assumes that the GCRs are a mix of WR wind material and material with Solar System composition [2]. There are two free parameters in their models, the mixing fraction of WR and solar material (p), and the WR star metallicity. They have performed calculations for various effective metallicities of the WR stars for both non-rotating and rotating stars. However, their calculations for rotating stars at present [38] do not extend to elements heavier than $\mathrm{Mg}$. In Figure 4 we show their WR model predictions for a WR star without rotation with an initial mass of $60 \mathrm{M}_{\mathrm{o}}$ and solar metallicity compared with the CRIS neon, iron, and nickel isotope ratios. The enhancement factors calculated by Meynet et al. [38] for individual isotopes have been used to calculate the mixing fraction required to obtain $a^{22} \mathrm{Ne} /{ }^{20} \mathrm{Ne}$ ratio normalized to 5.0, which is our CRIS measured value. This normalization yields a mixing fraction of $\mathrm{p}=0.052$. Comparing the predicted isotope ratios for iron and nickel with this mixing fraction to the derived CRIS abundance ratios results in generally good agreement, as shown in Figure 4, although the error bars for the rarest isotopes, e.g. ${ }^{61} \mathrm{Ni} /{ }^{58} \mathrm{Ni}$, are rather large.

We have also calculated the required mixing fraction using the model results for WR stars with $60 \mathrm{M}_{\mathrm{o}}$ and solar metallicity rotating at an initial velocity of $300 \mathrm{~km} / \mathrm{s} \mathrm{[38].}$ Rotation has a strong effect on the WR wind

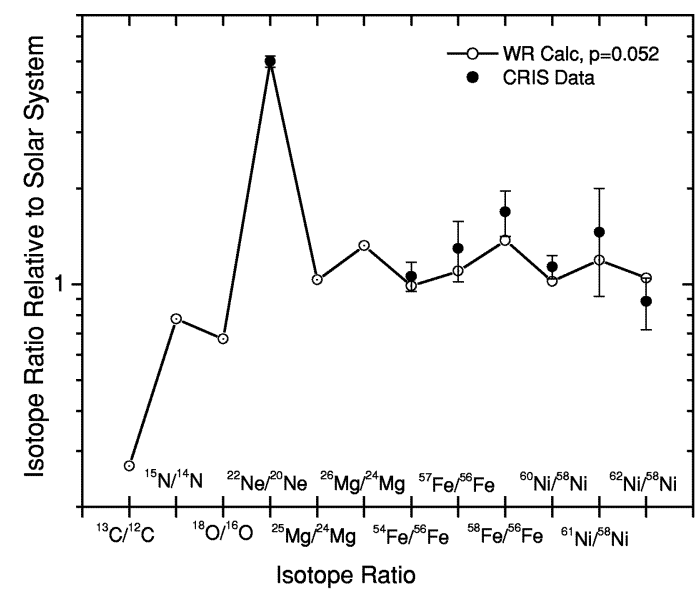

Figure 4-Isotopic ratios for iron and nickel isotopes are compared to a recent Wolf-Rayet modeling calculation (without rotation) [38]. The WR calculation is normalized to the ${ }^{22} \mathrm{Ne} /{ }^{20} \mathrm{Ne}$ ratio measured by CRIS.

composition and on the fraction of the stellar lifetime that the star spends in the WR and WC phases. Normalizing as before to the measured ${ }^{22} \mathrm{Ne} /{ }^{20} \mathrm{Ne}$ ratio of 5.0 we calculate a required mixing ratio $\mathrm{p}=0.215$ which seems quite large. The predicted isotope ratios are not plotted in Figure 4 since, at present, the calculations have not been extended above ${ }^{27} \mathrm{Al}$. Thus we see that a model that is intended to be more realistic than those with no rotation appears to make it more difficult to provide the observed ${ }^{22} \mathrm{Ne} /{ }^{20} \mathrm{Ne}$ enhancement. As noted by Meynet et al [38], these calculations are for a "typical" WR star with an initial mass of $60 \mathrm{M}_{\mathrm{o}}$. Further theoretical work is needed to obtain predictions which include contributions from the full range of WR stars in the Galaxy.

Additionally, Soutoul and Legrain [14,15] have developed a two-component model that incorporates diffusion of cosmic rays, in which GCRs come preferentially from the inner Galaxy. In this model the overabundance at Earth of ${ }^{22} \mathrm{Ne}$ results from a ${ }^{22} \mathrm{Ne}$ galactocentric radial gradient that is steeper than for the "main" cosmic ray component. (This "main" component in [15] is represented by Oxygen. ${ }^{20} \mathrm{Ne}$ would also be part of this main component). They present calculations [15] for 2 $\mathrm{GeV} /$ nuc neon that can produce the observed ${ }^{22} \mathrm{Ne}$ excess for large ${ }^{22} \mathrm{Ne}$ gradients, provided that there is also a moderate gradient for the main component. Their modeling calculations in [14] show that the ${ }^{22} \mathrm{Ne}$ overabundance shows a weak energy dependence, decreasing slightly from $2 \mathrm{GeV} /$ nuc to $1 \mathrm{GeV} /$ nuc. Although their calculations did not extend down to the CRIS energy range, the weak energy dependence of their calculation suggests that their model can be made consistent with the observed ${ }^{22} \mathrm{Ne}$ excess in the cosmic rays.

\section{Summary}

In summary, our measurements have led to an improved value for the ${ }^{22} \mathrm{Ne} /{ }^{20} \mathrm{Ne}$ source abundance ratio 
that is a factor of $5.0 \pm 0.2$ greater than for the solar wind. This ratio is significantly larger than any other available sample of "cosmic" matter with the exception of meteoritic Neon-E. For the two component WR model [38] (with no rotation) described above, a mixing fraction of about $5 \%$ is required to account for the ${ }^{22} \mathrm{Ne} /{ }^{20} \mathrm{Ne}$ source abundance ratio, and for that model the ratios of iron and nickel isotopes derived from CRIS data show good agreement within the measurement errors.

The diffusive two-component model $[14,15]$ can also produce the observed ${ }^{22} \mathrm{Ne} /{ }^{20} \mathrm{Ne}$ overabundance.

\section{Acknowledgments}

This research was supported by the National Aeronautics and Space Administration at Washington University, the California Institute of Technology (under grant NAG5-6912), the Jet Propulsion Laboratory, and the Goddard Space Flight Center. It was also supported by the McDonnell Center for the Space Sciences at Washington University.

\section{References}

1. Geiss, J., Solar Wind Composition and Implications about the History of the Solar System, Proc. of the 13th International Cosmic Ray Conference, 5, 3375, 1973.

2. Anders, E, and N. Grevesse, Abundances of the elements: Meteoritic and solar, Geochim. et Cosmochim. Acta 53, 197, 1989.

3. Maehl, R., F.A. Hagen, A.J. Fisher, J.F. Ormes, Astrophysical Implications of the Isotopic Composition of Cosmic Rays, Proc. of the 14th International Cosmic Ray Conference, 1, $367,1975$.

4. Garcia-Munoz, M., J.A. Simpson, and J.P. Wefel, The Isotopes of Neon in the GCRs, ApJ, 232, L95, 1979.

5. Wiedenbeck, M.E., and D.E. Greiner, Isotopic Anomalies in the Galactic Cosmic Ray Source, Phys. Rev. Lett., 46, 682, 1981.

6. Mewaldt, R.A., J.D. Spalding, E.C. Stone, and R.E. Vogt, High Resolution Measurements of Galactic Cosmic-Ray Neon, Magnesium, and Silicon Isotopes, ApJ 235, L95, 1980.

7. Lukasiak, A., P. Ferrando, F.B. McDonald, and W.R. Webber, Cosmic-Ray Isotopic Composition of $\mathrm{C}, \mathrm{N}, \mathrm{O}, \mathrm{Ne}, \mathrm{Mg}, \mathrm{Si}$ Nuclei in the Energy Range 50-200 MeV per Nucleon Measured by the Voyager Spacecraft During the Solar Minimum Period, ApJ, 426, 366, 1994.

8. Connell, J.J., and J.A. Simpson, High Resolution Measurements of the Isotopic Composition of GCR C, N, O, Ne, Mg, and $\mathrm{Si}$ from the Ulysses HET, Proc. of the 25th International Cosmic Ray Conference, 3, 381, 1997.

9. DuVernois, M.A., M. Garcia-Munoz, K.R. Pyle, J.A. Simpson, and M.R. Thayer, The Isotopic Composition of Galactic Cosmic-Ray Elements from Carbon to Silicon: The Combined Release and Radiation Effects Satellite Investigation, ApJ, 466, 457,1996.

10. Woosley, S.E., and T.A. Weaver, Anomalous Isotopic Composition of Cosmic Rays, ApJ, 243, 651,1981.

11. Wiedenbeck, et al., Constraints on the Nucleosynthesis of Refractory Nuclides in Galactic Cosmic Rays, this conference.

12. Olive, K.A., and D.N. Schramm, OB Associations and the Nonuniversality of the Cosmic Abundances: Implications for Cosmic Rays and Meteorites, ApJ, 257, 276, 1982.
13. Cassé, M., and J.A.Paul, On the Stellar Origin of the ${ }^{22} \mathrm{Ne}$ Excess in Cosmic Rays, ApJ, 258, 860, 1982.

14. Soutoul, A., and Legrain, R., Galactic source distribution of heavy cosmic rays and the energy dependent overabundance of ${ }^{22} \mathrm{Ne}$, Proc. of the 26th International Cosmic Ray Conference, 4, 180, 1999.

15. Soutoul, A., and Legrain, R., ${ }^{22} \mathrm{Ne}$ excess in cosmic rays from the inner Galaxy, in Acceleration and Transport of Energetic Particles Observed in the Heliosphere: ACE 2000 Symposium, Ed Richard A. Mewaldt, et al., AIP Conference Proceedings 528, 417, 2000.

16. Van der Hucht, K.A., B. Hidayat, A.G. Admiranto, K.R. Supelli, and C. Doom, The Galactic Distribution and Subtype Evolution of Wolf-Rayet Stars. III, Astron. Astrophys., 199, 217,1988

17. Meynet, G., and A. Maeder, WR Stars and Isotopic Anomalies in Cosmic Rays, Adv. Space Res., 19, 763, 1997.

18. Maeder, A. and G. Meynet, Isotopic anomalies in cosmic rays and the metallicity gradient in the Galaxy, Astron. Astrophys., 278, 406, 1993.

19. Mewaldt, R.A., The Abundances of Isotopes in the Cosmic Radiation, in Cosmic Abundances of Matter, Ed. By C.J. Waddington, AIP Conference Proceedings 183, 124, 1989.

20. Stone, E.C., C.M.S. Cohen, W.R. Cook, A.C. Cummings, B. Gauld, et al., The Cosmic-Ray Isotope Spectrometer for the Advanced Composition Explorer, Space Science Reviews, 86, $285,1998$.

21. Stone, E.C., and Wiedenbeck, M.E., A Secondary Tracer Approach to the Derivation of Galactic Cosmic-Ray Source Isotopic Abundances, ApJ, 231, 606, 1979.

22. Binns, W.R., et al. (2001), Galactic Cosmic Ray Neon Isotopic Abundances Measured by the Cosmic Ray Isotope Spectrometer (CRIS) on ACE, Advances in Space Research, 27, 767, 2001

23. Binns, W.R., M.E. Wiedenbeck, E.R. Christian, A.C. Cummings, J.S. George, et al., Galactic Cosmic Ray Neon Isotopic Abundances Measured on ACE: in Acceleration and Transport of Energetic Particles Observed in the Heliosphere, ACE 2000 Symposium, Ed. R.A. Mewaldt et al., AIP Conference Proceedings 528, 413, 2000.

24. Leske, R.A, R.A. Mewaldt, A.C. Cummings, J.R. Cummings, E.C. Stone, et al., The Isotopic Composition of Anomalous Cosmic Rays from SAMPEX, Space Sci. Revs. 78, 149, 1996.

25. Leske, R.A., R.A. Mewaldt, E.R. Christian, C.M.S. Cohen, A.C. Cummings, et al., Measurements of the Isotopic Composition of Anomalous Cosmic Ray N, O, and Ne from ACE, Proc. of the 26th International Cosmic Ray Conference, 7, 539, 1999.

26. Leske, R.A., R.A. Mewaldt, C.M.S. Cohen, A.C. Cummings, E.C. Stone, et al., Event-to-event variations in the isotopic composition of neon in solar energetic particle events, GRL, 26, 2693, 1999.

27. Ozima, M., and Podosek, F.A., Noble Gas Geochemistry, Cambridge: Cambridge University Press, 1983.

28. Kehm, K., Thesis, Washington University, 2000.

29. Amari, S., R.S. Lewis, and E. Anders, Interstellar Grains in Meteorites: III. Graphite and its Noble Gases, Geochim. et Cosmochim. Acta, 59, 1411, 1995.

30. Cohen, C.M.S., R.A. Leske, E.R. Christian, A.C. Cummings, R.A. Mewaldt, P.L. Slocum, E.C. Stone, T.T. von Rosenvinge, and M.E. Wiedenbeck, in Acceleration and Transport of Energetic Particles Observed in the Heliosphere: ACE 2000 Symposium, Ed Richard A. Mewaldt, et al., AIP Conference Proceedings 528, 55, 2000.

31. Leske. R.A., R.A. Mewaldt, E.R. Christian, C.M.S. Cohen, A.C. Cummings, P.L. Slocum, E.C. Stone, T.T. von 
Rosenvinge, and M.E. Wiedenbeck, in Acceleration and Transport of Energetic Particles Observed in the Heliosphere: ACE 2000 Symposium, Ed Richard A. Mewaldt, et al., AIP Conference Proceedings 528, 293, 2000.

32. Huss, G.R. and R.S. Lewis, Noble gases in presolar diamonds I: Three distinct components and their implications for diamond origins, Meteoritics, 29, 791, 1994.

33. Weiler, R, H. Baur, and P. Signer, Noble gases from solar energetic particles revealed by closed system stepwise etching of lunar soil minerals, Geochim. et Comochim. Acta, 50, 1997, 1986.

34. Wielen, R., Fuchs, B., and C. Dettbarn, On the Birth-place of the Sun and the Places of Formation of other Nearby Stars, Astron. Astrophys., 314, 438, 1996.

35. Lewis, R.S., S. Amari, and E. Anders, Interstellar grains in meteorites: II. $\mathrm{SiC}$ and its noble gases, Geochim. et Cosmochim. Acta, 58, 471, 1994.

36. Chiosi, C, and A. Maeder, The Evolution of Massive Stars, Ann. Rev. Astron. Astrophys, 24, 329, 1986.

37. Nugis, T., P.A. Crowther, and A.J. Willis, Clumping-corrected mass-loss rates of Wolf-Rayet stars, Astron. Astrophys. 333, 956, 1998.

38. Meynet, G., M. Arnould, G. Paulus, and A. Maeder, WolfRayet Star Nucleosynthesis and the Isotopic Composition of the Galactic Cosmic Rays, Space Sci. Rev., 2000 To be published, and Private Communications.

39. Wiedenbeck, M.E. et al., The Origin of Primary Cosmic Rays: Constraints from ACE Elemental and Isotopic Composition Observations, Space Sci. Rev., 2001, Accepted for publication. 